\title{
1 Preclinical and Toxicology Studies of BRD5529, a Selective Inhibitor of CARD9
}

2

3

$6{ }^{1}$ Thoracic Diseases Research Unit, Departments of Medicine and Biochemistry, Mayo

7 Clinic, Rochester, Minnesota, 55905 USA, ${ }^{2}$ Department of Laboratory Medicine and

8 Pathology, Mayo Clinic, Rochester, Minnesota, 55905

9 *Address correspondence and reprint requests to Dr. Theodore Kottom, 8-23 Stabile, Mayo

10 Clinic, Rochester, MN 55905; Tel.: (507) 284-8418; Fax: (507) 284-5421; E-mail address:

11 kottom.theodore@mayo.edu

Keywords: CARD9, BRD5529, Pneumocystis, Inflammation

Abbreviations: CARD9 (Caspase recruitment domain-containing protein 9), PCP (Pneumocystis pneumonia), IP (Intraperitoneally), (IACUC) Institutional Animal Care and Use Committee, CLR (C-type lectin receptor), (H\&E) Hematoxylin and eosin, HAART (highly active antiretroviral therapy), (TMP-SMX) trimethoprim-sulfamethoxazole, (PJP) Pneumocystis jirovecii pneumonia.

\section{ABSTRACT}

Background: Exuberant inflammation during Pneumocystis pneumonia leads to lung injury.

CARD9 is a central mediator of inflammatory signaling mediated by C-type lectin receptors.

25 CARD9 inhibitor BRD5529 has been shown to be an effective in vitro inhibitor of Pneumocystis $\beta$-glucan-induced proinflammatory signaling and downstream TNF-alpha 
27 production, suggesting its viability as a candidate for preliminary drug testing as an anti-

28 inflammatory agent in the rodent Pneumocystis pneumonia model (PCP).

29 Methods: To assess for potential toxicity, mice were injected intraperitoneally (IP) daily either

30 with vehicle or BRD5529 at $0.1 \mathrm{mg} / \mathrm{kg}$ or $1.0 \mathrm{mg} / \mathrm{kg}$ for two weeks. Mouse weights were taken

31 daily. At day 14, mice were euthanized, weighed, and analyzed by flexiVent ${ }^{\mathrm{TM}}$ for lung

32 stiffness. Lungs, liver, and kidney were then harvested for H\&E staining and pathology

33 scoring. Lung samples were further analyzed for proinflammatory cytokines via ELISA and

34 extracellular matrix generation via quantitative PCR (q-PCR). Blood collection postmortem

35 was performed for blood chemistry analysis.

36 Results: BRD5529 at both doses of IP administration resulted in no significant changes in

37 daily or final weight gain. Analysis of lung stiffness by flexiVent ${ }^{\mathrm{TM}}$ showed no significant

38 differences between the control or treated groups. Furthermore, ELISA results for

39 proinflammatory IL-1 Beta, IL-6, and TNF-alpha showed no major differences in the

40 respective groups. qPCR analysis of extracellular matrix transcripts collagen type I, alpha 1

41 (Col1a1) and fibronectin $(F n)$ were statically similar as well in the treated and control groups.

42 Examination and pathology scoring of H\&E slides from lung, liver, and kidney from the each

43 of the mice in all groups and subsequent pathology scoring showed no significant change.

44 Blood chemistry analysis revealed similar, non-significant patterns.

45 Conclusions: BRD5529 in our initial general safety and toxicology assessments displayed no

46 inherent safety concerns in the analyzed parameters. These data support broader in vivo

47 testing of the inhibitor as a timed adjunct therapy to the deleterious proinflammatory host

48 immune response often associated with anti-Pneumocystis therapy.

\section{Introduction}

51 Caspase recruitment domain-containing protein 9 (CARD9) is a central mediator

52 downstream of C-type lectin receptors (CLRs) that is vital for microbial pathogen 
53 proinflammatory host immune response and organism burden control [1]. CARD9 is highly

54 expressed in myeloid cells and shown to be particularly important in fungal infections [2].

55 Others have demonstrated CARD9 pathway intervention with the chemical CARD9 inhibitor

56 BRD5529 can directly mimic a protective variant of the protein and may provide therapeutic

57 benefit with those with inflammatory bowel disease [3]. Recently, we have shown that

58 preincubation of BRD5529 with RAW macrophages prior to the application of

59 proinflammatory $\beta$-glucans from the lung pathogen Pneumocystis spp., results in substantial

60 reduction in downstream CARD9 proinflammatory signaling and subsequent TNF-alpha

61 release, suggesting that timed therapeutic intervention during or after anti-Pneumocystis

62 treatment, may greatly improve the deleterious effects on the host caused by organism

63 killing and release of proinflammatory carbohydrates [4]. The purpose of this study was to

64 evaluate the short-term administration of CARD9 inhibitor BRD5529 in mice via IP

65 administration and address potential detrimental responses to the inhibitor via physiological,

66 inflammatory, and toxicological analysis. These data demonstrate the safety of BRD5529

67 and support broader clinical development of the CARD9 inhibitor for in vitro administration

68 in the PCP mouse treatment model as a therapeutic tool to treat PCP host inflammation

69 during anti-Pneumocystis treatment.

71 2. Methods

72 2.1. Animals

73 Equal numbers of male and female C57BL/6 mice (The Jackson Laboratory) at 10-

7412 weeks of age were used for all experiments. All animal procedures were performed in 75 accordance with the Laboratory Animal Welfare Act, the Guide for the Care and Use of 
76 Laboratory Animals Welfare Act, and the Mayo Clinic Institutional Animal Care and Use

77 Committee (IACUC) (Approval number: A00005722-20).

\subsection{Administration of BRD5529}

80

BRD5529 was obtained for Sigma Aldrich. Due to the lack of solubility of the inhibitor

82 in water or saline, the inhibitor was prepared with $1 \%$ Methocel $^{\mathrm{TM}}$ [5]. Intraperitoneal

83 treatment $(100 \mu \mathrm{l})$ with $1 \%$ Methocel $^{\mathrm{TM}}$ (vehicle, control mice group) or the indicated

84 concentration $(\mathrm{mg} / \mathrm{kg})$ of the BRD5529 inhibitor in Methocel ${ }^{\mathrm{TM}}$ was initiated on day 0 and

85 subsequentially every day for 14 days. At day 14, mice were sacrificed, and subsequent

86 analysis performed as described below.

2.3. Flexivent ${ }^{\mathrm{TM}}$ analysis

FlexiVent ${ }^{\mathrm{TM}}$ analysis was performed at described previously [6].

\subsection{ELISA determination of cytokine release.}

92 Cytokines were analyzed from total lung homogenates. ELISA kits to measure

93 mouse IL-1 Beta ,IL-6, and TNF-alpha were purchased from Thermo Fisher Scientific.

\subsection{Quantitative polymerase chain reaction analysis}

To extract RNA from mouse lung, tissue was lysed and homogenized with Buffer RLT

98 Plus (supplied with the RNeasy ${ }^{\circledR}$ Plus Mini Kit; Qiagen). The lysate was passed through a

99 genomic DNA eliminator spin column, ethanol was added, and the sample was applied to a

100 RNeasy MinElute spin column according to the manufacturer's instructions. An iScript ${ }^{\mathrm{TM}}$

101 Select cDNA synthesis kit (Bio-Rad) was used for reverse transcription using oligo (dT) 
102 primers and random hexamer primer mix. A SYBR green PCR kit (Bio-Rad) was used for

103 quantitative real-time PCR and was performed and analyzed on a CF96 Touch ${ }^{\mathrm{TM}}$ Real-Time

104 PCR Detection System (Bio-Rad). The sequences of the primer pairs are listed in

105 Supplementary Table 1.

106

107 2.6. Biochemical analysis

108 For blood chemistry analysis, serum was analyzed with the Piccolo Xpress $^{\mathrm{TM}}$

109 Chemistry Analyzer according to the manufacturer's instructions.

110

111 2.7. Histology analysis

112 For histological analysis, lung, liver, and kidney samples were fixed in 10\% neutral formalin.

113 Paraffin embedding and staining were performed at the Mayo Clinic Histology Core,

114 Scottsdale, AZ. Sections $(5 \mu \mathrm{m})$ were stained with Hematoxylin and eosin (H\&E) and

115 graded blindly for the extent of organ inflammation by a Mayo Pathologist. The sections

116 were scored as follows: 1+, mild perivascular aggregates; 2+, heavy perivascular

117 aggregates; 3+, mild alveolar aggregates; 4+, alveolar exudate and heavy alveolar

118 aggregates; and 0, normal. These scores were based upon grading of the entire organ

119 surface area present on the slide section.

120

\section{2.8. Statistical Analysis}

122 For multigroup data, initial analysis was first performed with analysis of variance

123 (ANOVA) to determine overall different differences. If ANOVA indicated overall differences,

124 subsequent group analysis was then performed by 2-sample unpaired Student's $t$-test for 125 normally distributed variables. Evaluation of data was conducted using Prism 9 for macOS, 126 version 9.1.0 (GraphPad). Values of $p<0.05$ were considered significant. 


\subsection{BRD5529 IP administration resulted in no significant weight loss}

A

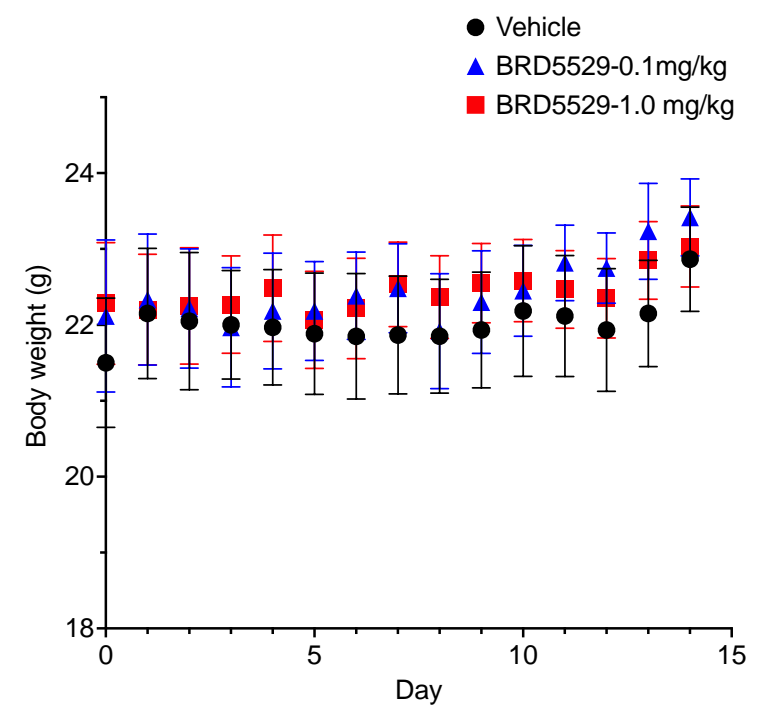

B

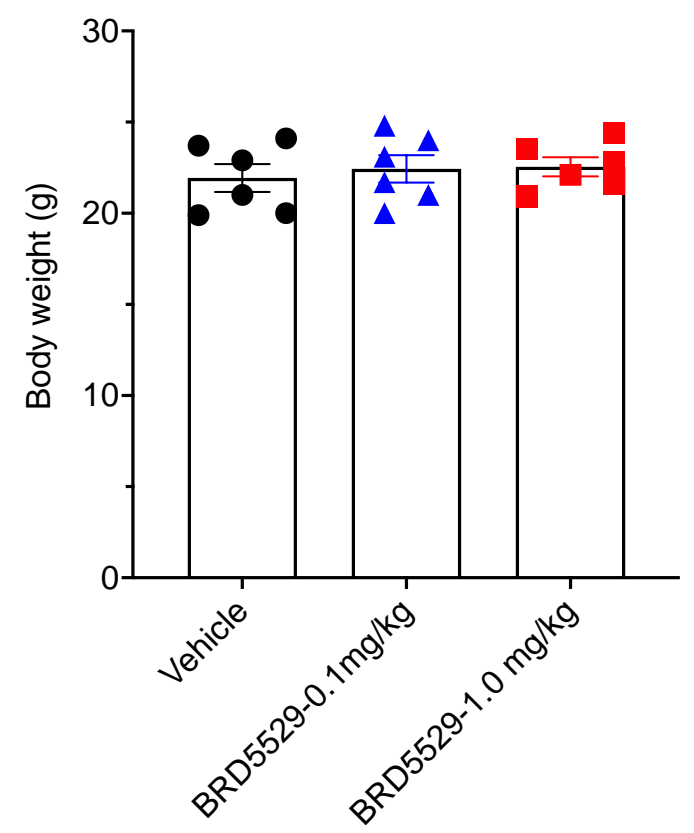

131

132 Fig. 1. Effects of mouse body weight with IP administration daily of BRD5529. (A)

133 Shows the daily weight changes in the vehicle control versus the $0.1 \mathrm{mg} / \mathrm{kg}$ and $1.0 \mathrm{mg} / \mathrm{kg}$

134 doses of IP administered BRD5529 daily for 14 days. (B) Shows the final weights for the 3

135 groups after 14 days. ( $n=6$ mice/group). No significant weight changes were noted between

136 the three groups.

137

$138 \quad$ 3.2. Static lung compliance after BRD5529 IP administration

To measure lung function in mice after 14 days of IP administration of vehicle, or

140 BRD5529 at $0.1 \mathrm{mg} / \mathrm{kg}$ or $1.0 \mathrm{mg} / \mathrm{kg}$ we used the flexiVent ${ }^{\mathrm{TM}}$ apparatus. Daily IP injections 
141 of all three conditions for 14 days resulted in no significant increases in lung static compliance

142 between the three groups tested (Fig 2).

143

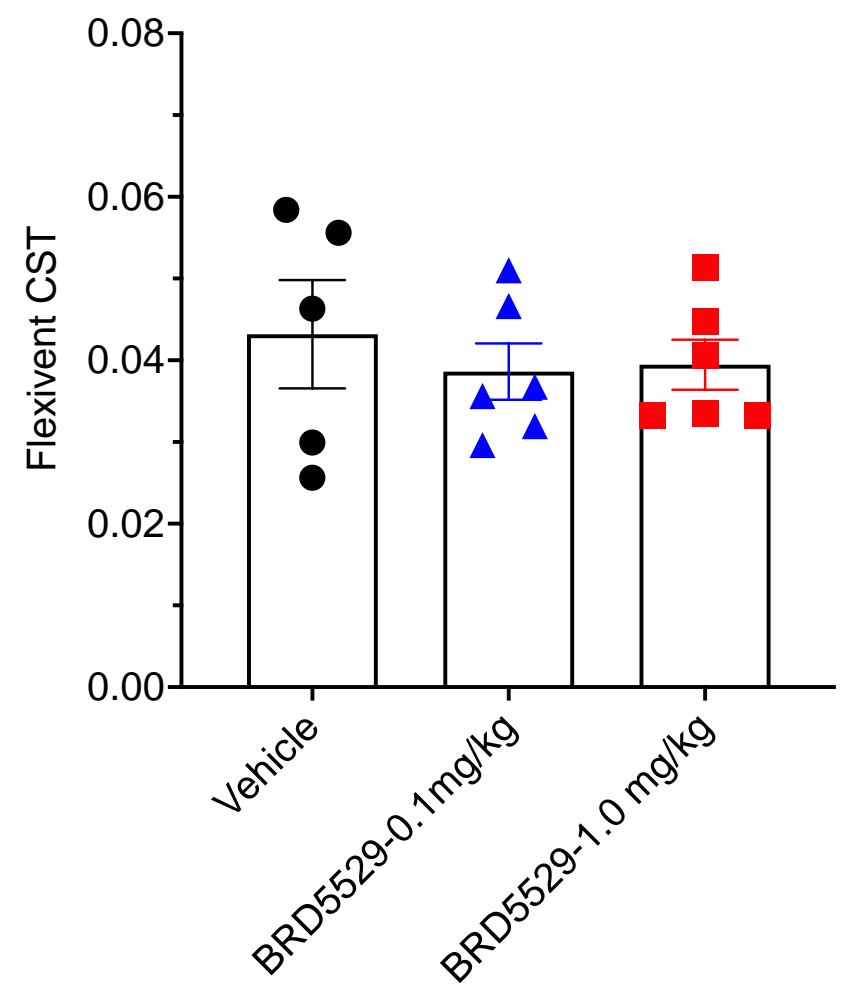

145 Fig. 2. CARD9 inhibitor BRD5529 effects on lung compliance. Lung quasi-static

146 compliance (CST; reflects the intrinsic elastic properties of the lung and chest at rest). $(n=$

1476 mice/group). No significant changes in CST were noted between the three groups.

149 3.3. Measurements of lung cytokines in BRD5529 IP administrated and control groups

150 The production of inflammatory cytokines IL-1 Beta, IL-6, and TNF-alpha were

151 measured in whole lung lysates and as shown in (Fig. 3A-C). No significant alterations were 152 noted from the vehicle control. 
A

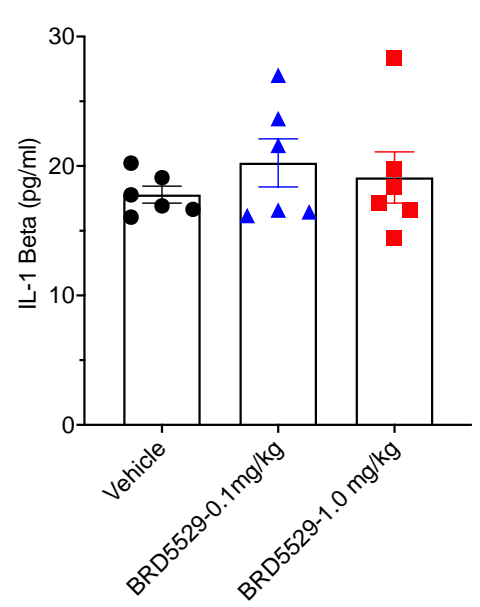

B

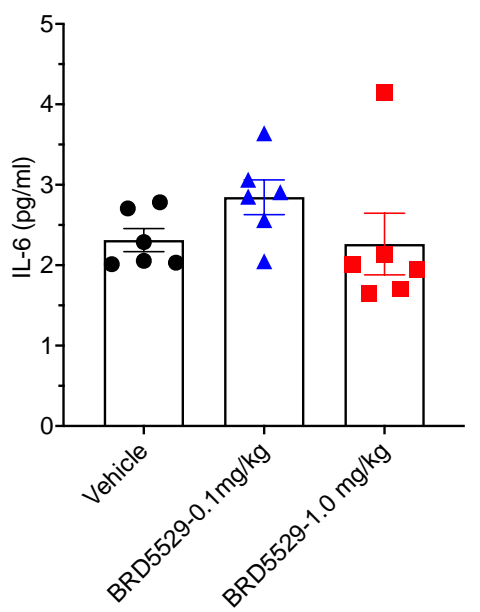

C

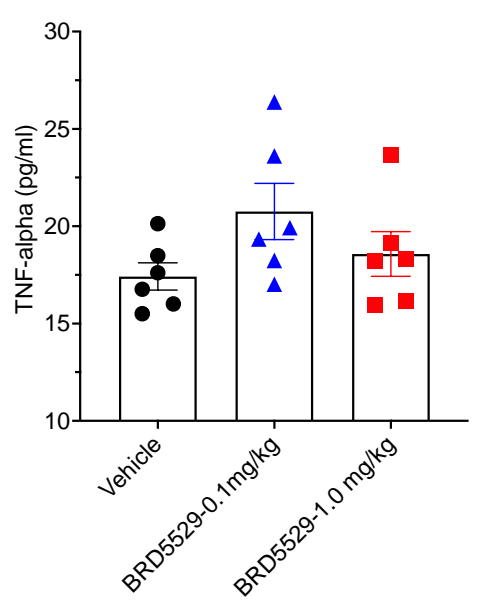

154 Fig. 3. CARD9 inhibitor BRD5529 effects on lung proinflammatory cytokine

155 production. (A) IL-1 Beta, (B) IL-6, and (C) TNF-alpha production was measured from total

156 lung lysates from day 14 of the experiment. ( $n=6$ mice/group). No significant differences

157 were noted between the groups.

159 3.4. Analysis of mRNA extracellular matrix generation

160 qPCR was implemented to determine the levels of mRNA expression of Collagen

161 Type Alpha 1 Chain (Col1a1) and Fibronectin ( $F n)$, both extracellular matrix related genes

162 used as markers for profibrotic development [7]. Beta 2 Microglobulin (B2M) was used as a

163 housekeeping gene. As shown in (Fig. 4A-B), no significant differences were noted in the 3

164 groups at day 14 in the respective lung samples. 
A

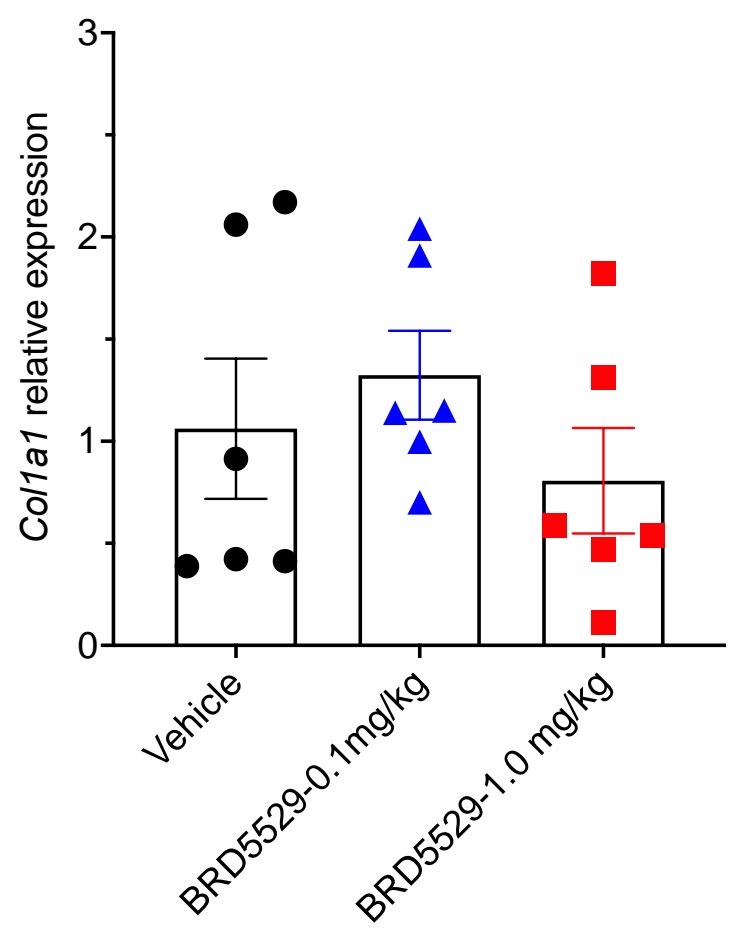

B

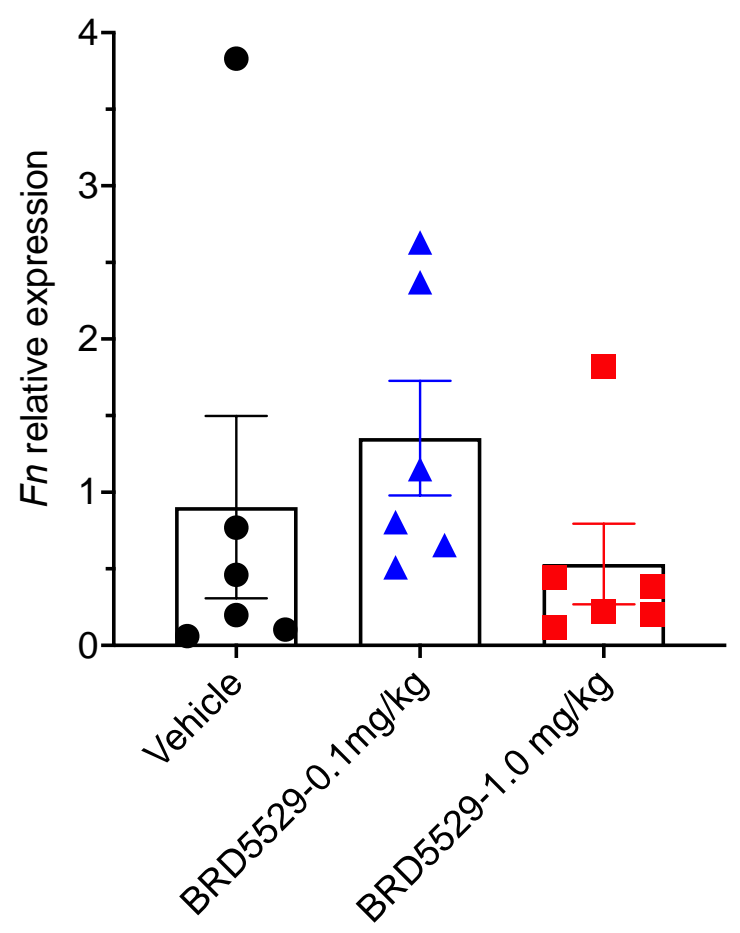

165

166

167

168

169

170

171

172

173

174

Fig. 4. Quantitation of Col1a1 and Fn mRNA in total lung RNA after vehicle and BRD5529 administration for 14 days. Ratios of (A) Col1a1 and (B) Fn to B2M in total lung RNA. ( $\mathrm{n}=6$ mice/group). No significant differences were noted between the groups.

\subsection{Serum chemistry data}

Complete group mean serum chemistry data from data 14 are presented in (Fig. 5A-

N). There were no noteworthy changes in any either BRD5529 dose groups from the vehicle control. 
A

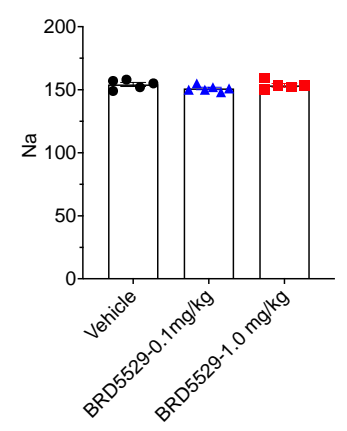

F

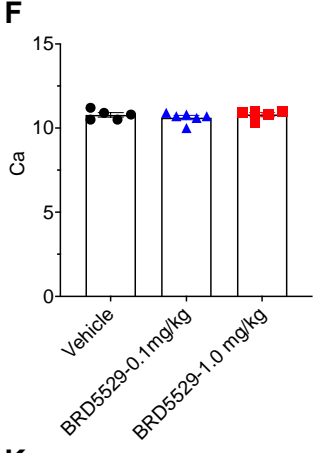

K

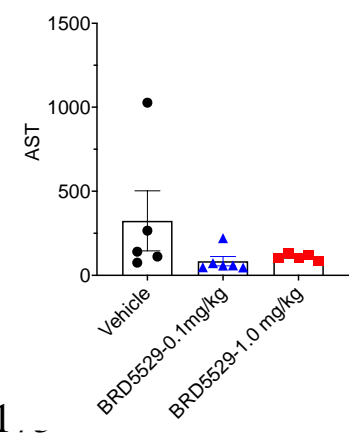

B

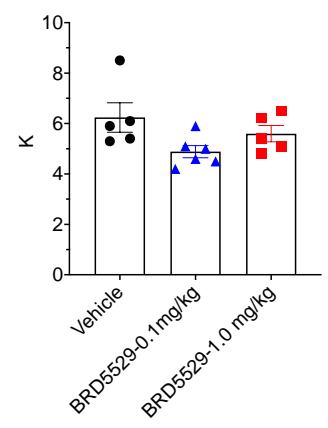

G

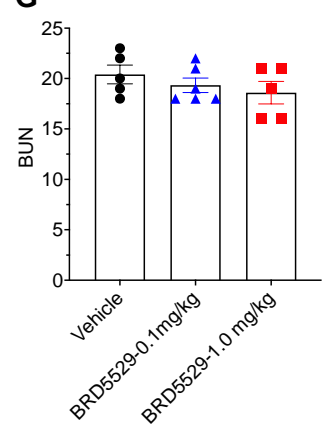

L

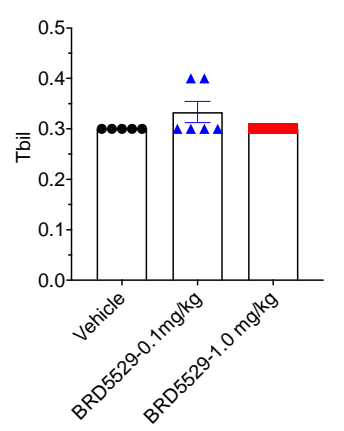

C

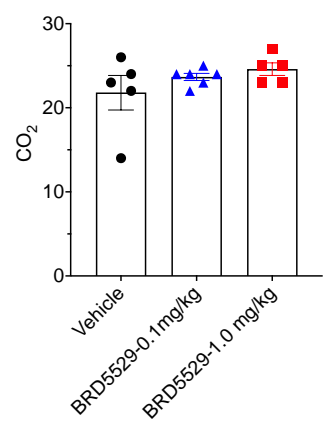

H

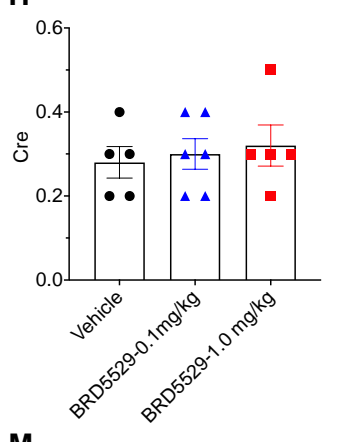

M

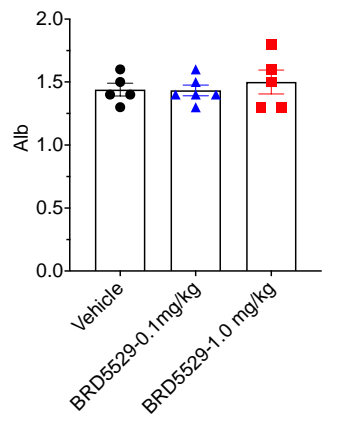

D

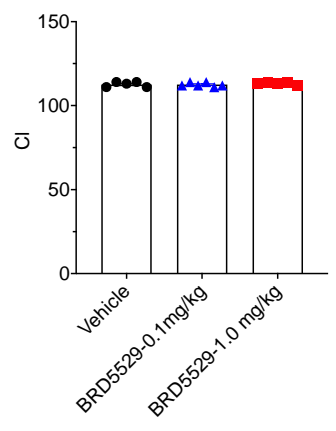

I

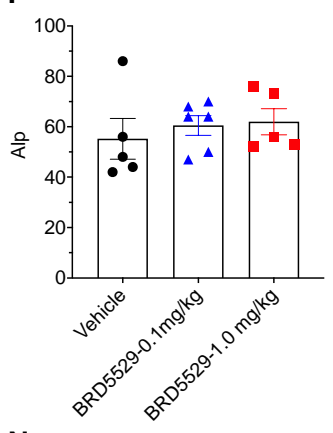

N

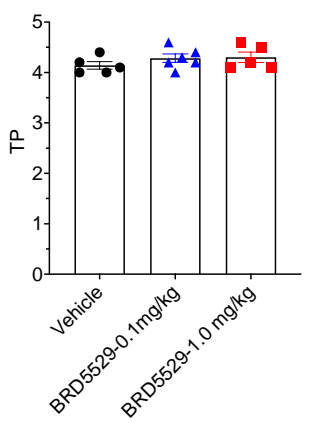

E

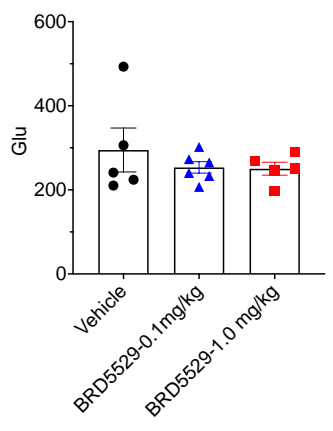

J

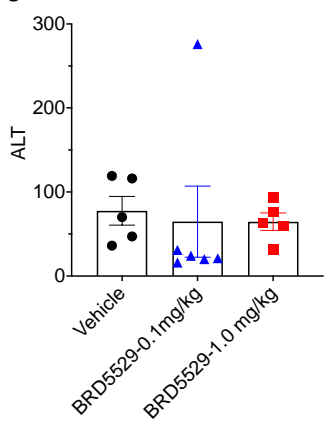

176 Fig. 5. Serum chemistry parameters. $\mathrm{Na}$, sodium (mmol/L); K, potassium (mmol/L); $\mathrm{CO}_{2}$,

177 carbon dioxide (mmol/L); Cl, chloride (mmol/L); Glu, glucose (mg/dL); Ca, Calcium (mg/dL);

178 BUN, blood urea nitrogen $(\mathrm{mg} / \mathrm{dL})$; Cre, creatine $(, \mathrm{g} / \mathrm{dL})$; Alp, alkaline phosphatase $(\mathrm{U} / \mathrm{L})$;

179 ALT, alanine aminotransferase (U/L); Tbil, total bilirubin (mg/dL); Alb, albumin $(\mathrm{g} / \mathrm{dL}) ; \mathrm{TP}$,

180 total protein $(\mathrm{g} / \mathrm{dL})$. ( $\mathrm{n}=5-6$ mice/group). No significant differences were noted between the

181 groups.

182

183 3.6. Histology analysis 
185 treated- and vehicle groups did not reveal any abnormality and all organs appear normal

186 (score 0 for all parameters).

187

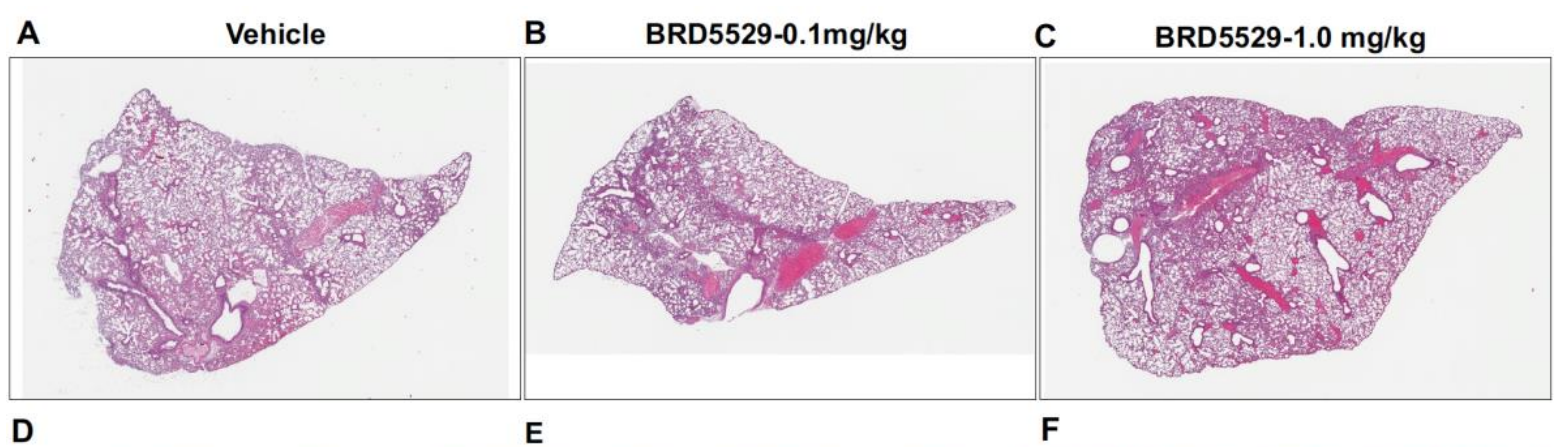

188
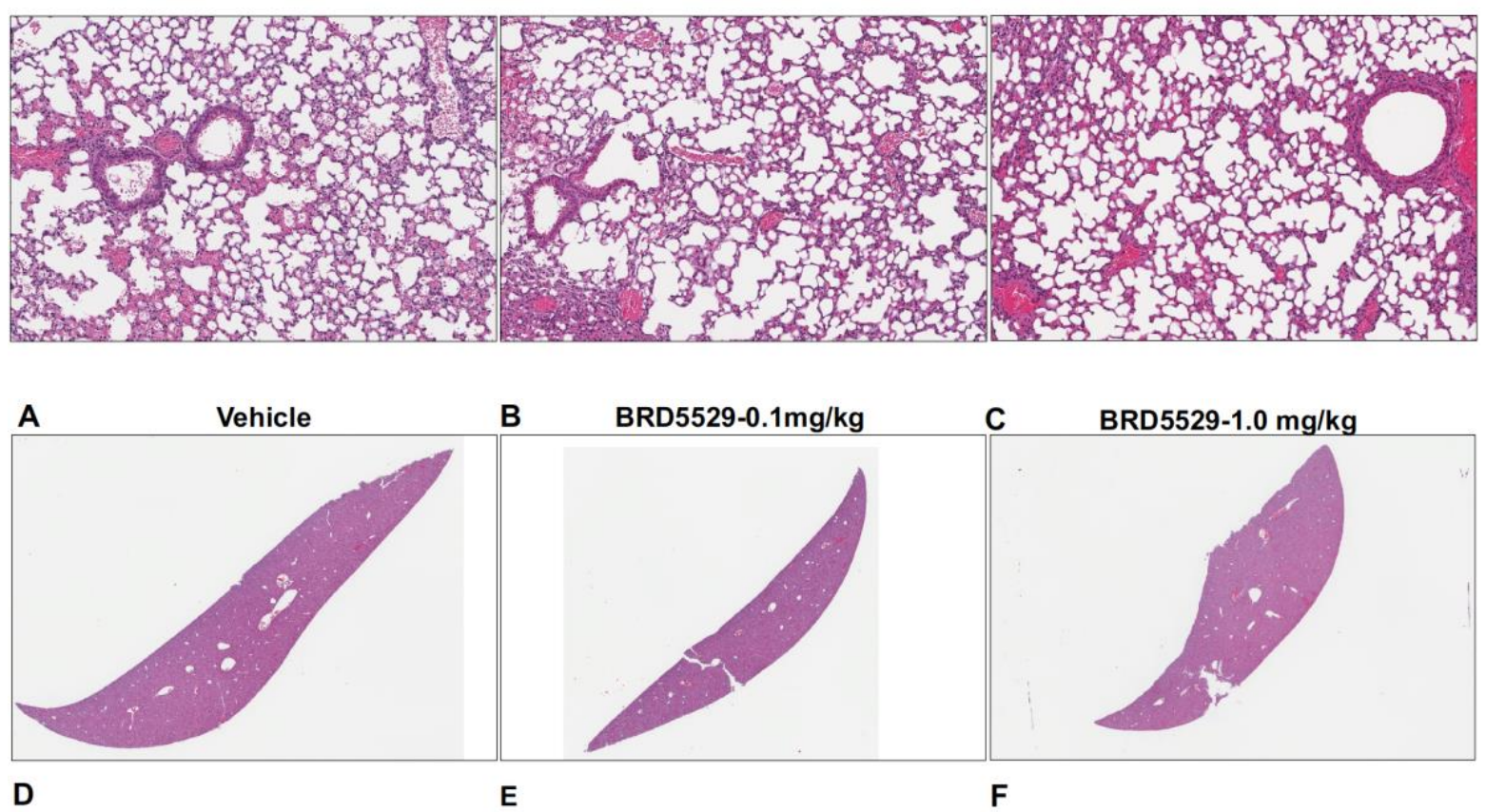

F

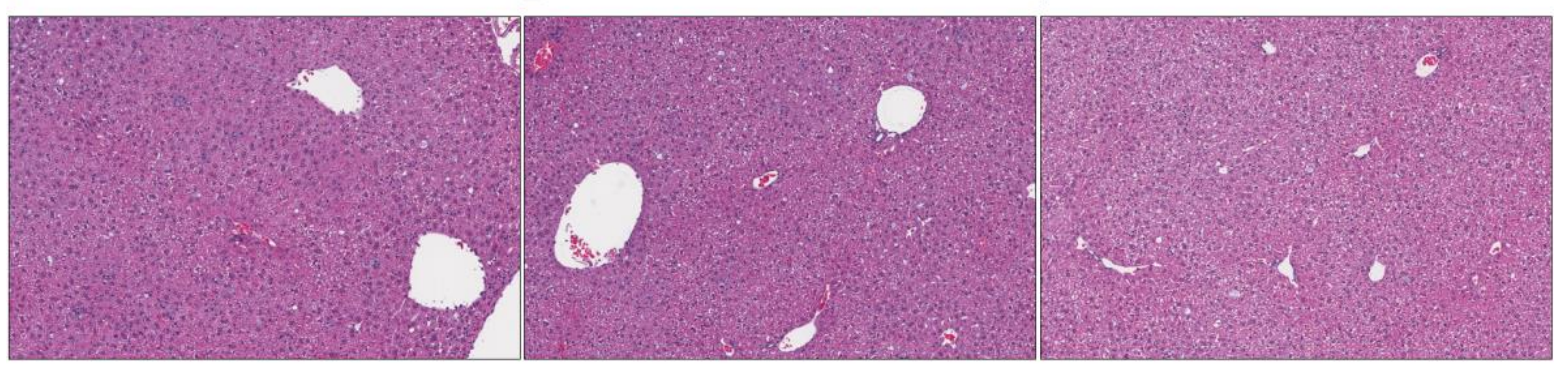




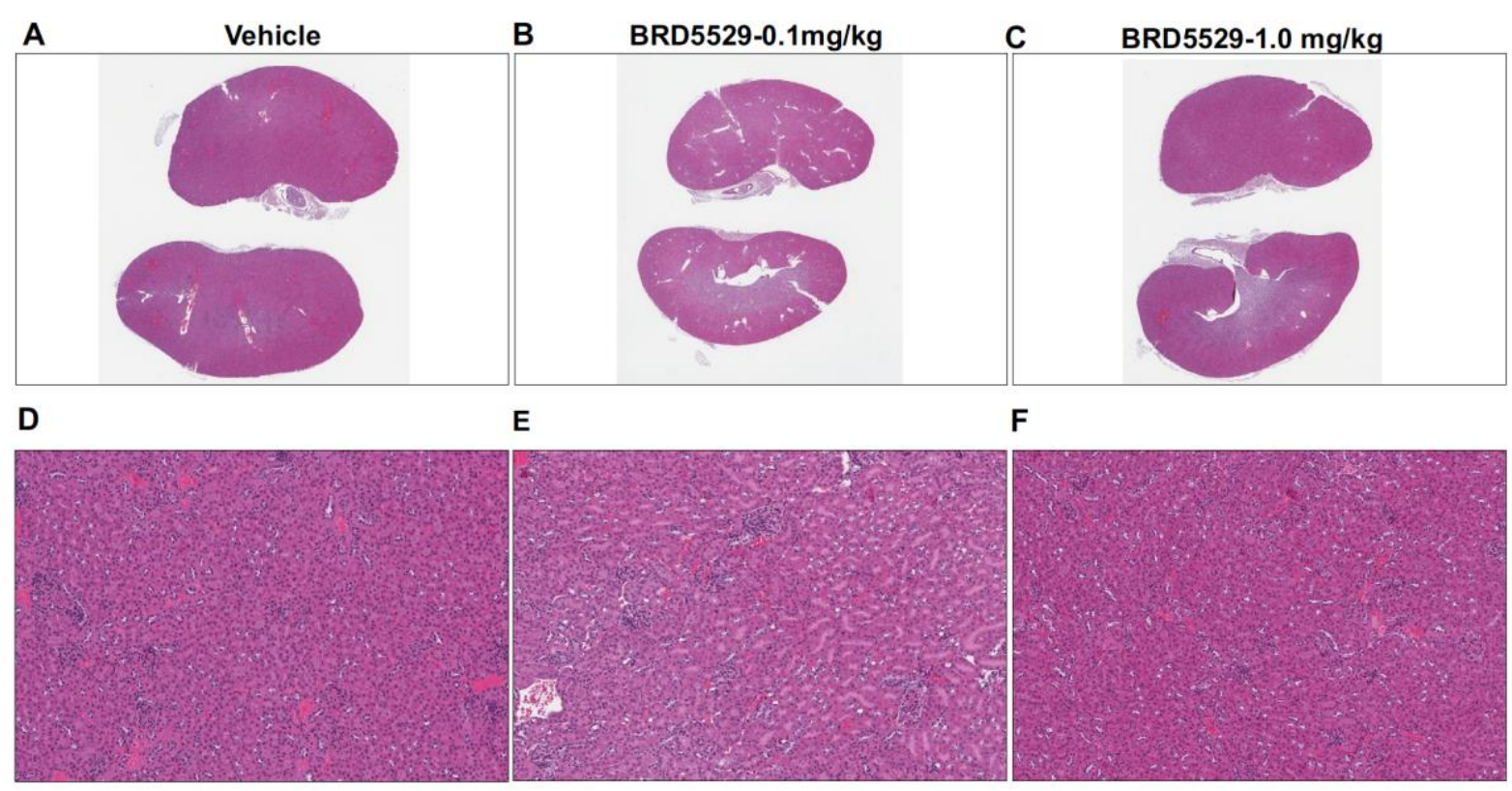

191 Fig. 6. Lung, liver, and kidney histopathology of 14-day IP treated vehicle or BRD5529

192 CARD9 inhibitor. Hematoxylin and eosin (H\&E) staining was performed on sections of lung

193 (top two panels), liver (middle two panels), and kidney (bottom two panels) from mice in all

194 groups. (A) Vehicle control. (B) BRD5529 at $0.1 \mathrm{mg} / \mathrm{kg}$. (C) BRD5529 at $1 \mathrm{mg} / \mathrm{kg}$. No

195 histological changes were present in lung, liver, and kidneys in the vehicle and BRD5529

196 doses tested.

\section{4. Discussion}

Fungi are major contributors to opportunistic infections in those with advanced HIV.

201 Pneumocystis jirovecii pneumonia (PCP), caused by Pneumocystis jirovecii is among the 202 most common pathogens in AIDS populations across the globe. Implementation of highly 203 active antiretroviral therapy (HAART) has decreased the overall incidence of PCP, but 204 the ability to receive this treatment is limited and cases of PJP requiring hospitalization is 205 still quite high [8]. 
The most current anti-Pneumocystis therapy, trimethoprim-sulfamethoxazole (TMP-SMX), has proven to an effective antimicrobial combination to treat Pneumocystis jirovecii pneumonia (PJP). Although effective, the exuberant inflammatory response following fungal cell death, via the exposure of newly exposed $\beta$-glucans can prove highly detrimental to the host [9-11]. Indeed, when corticosteroids are utilized in HIV patients with moderate-severe PJP, there is a significant decrease in mortality and morbidity noted [12]. In the non-HIV patient, data on adjuvant corticosteroids is less clear and no consensus has been determined. Even though steroids may be beneficial to the patient in these settings, there are still both short-term (co-infections, hyperglycemia) and long demonstrated that in vivo, macrophages pre-incubated with the caspase recruitment domain-

218 containing protein 9 (CARD9) inhibitor BRD5529 have significant reductions in their ability to

219 generate proinflammatory signaling and downstream TNF-alpha production upon stimulation

220 with Pneumocystis $\beta$-glucans [4]. These results lead us to hypothesize that BRD5529 may

221 be used in vivo as an adjunct therapy similar to corticosteroids [4]. As part of the development

222 toward human clinical application, a thorough preclinical assessment must be conducted to

223 evaluate safety and potential toxicity of the BRD5529 CARD9 inhibitor, and to the best of our

224 knowledge this is the first study conducted to examine this. The preclinical profile presented

225 here suggests thar BRD5529 could meet these early criteria.

226 In this study we gave mice BRD5529 either at $0.1 \mathrm{mg} / \mathrm{kg}$ or $1.0 \mathrm{mg} / \mathrm{kg}$ once a day for

22714 days via IP administration. In acute toxicity studies, administration of BRD5529 appeared

228 to be well tolerated in mice at both doses. Parameters such as weight loss, lung function, 229 lung-specific proinflammatory response, lung extracellular matrix mRNA generation, blood 
230 toxicology analysis, and H\&E histological examine of lung, liver, and kidney samples yielded

231 no significant changes as compared to the vehicle control.

232

\section{5. Conclusions}

Based on these preliminary findings, the use of BRD5529 in the future employing in

236 vivo mouse studies to determine whether the CARD9 inhibitor can be used to reduce the

237 deleterious effects of the host proinflammatory in the PCP model seems safe and feasible.

238

239 Funding

240 This work was supported by the Mayo Foundation; the Walter and Leonore 241 Annenberg Foundation, and NIH grant [R01-HL62150] to A.H.L.

243 CRediT authorship contribution statement

Theodore J. Kottom: Writing - original draft, drafted the manuscript, performed the experiments, performed data analysis. Kyle Schaefbauer: performed the experiments. Eva

M. Carmona: Writing - review and editing. Eunhee S. Yi: Writing - review and editing, design and analysis.

Declaration of competing interest

\section{References}


257 [1] J. Tang, G. Lin, W.Y. Langdon, L. Tao, J. Zhang, Regulation of C-Type Lectin Receptor-

258 Mediated Antifungal Immunity, Front Immunol 9 (2018) 123.

259 [2] R.A. Drummond, L.M. Franco, M.S. Lionakis, Human CARD9: A Critical Molecule of

260 Fungal Immune Surveillance, Front Immunol 9 (2018) 1836.

261 [3] E.S. Leshchiner, J.S. Rush, M.A. Durney, Z. Cao, V. Dancik, B. Chittick, H. Wu, A.

262 Petrone, J.A. Bittker, A. Phillips, J.R. Perez, A.F. Shamji, V.K. Kaushik, M.J. Daly, D.B.

263 Graham, S.L. Schreiber, R.J. Xavier, Small-molecule inhibitors directly target CARD9 and

264 mimic its protective variant in inflammatory bowel disease, Proc Natl Acad Sci U S A 114(43)

265 (2017) 11392-11397.

266 [4] T.J. Kottom, E.M. Carmona, A.H. Limper, Targeting CARD9 with Small-Molecule

267 Therapeutics Inhibits Innate Immune Signaling and Inflammatory Response to Pneumocystis

268 carinii beta-Glucans, Antimicrob Agents Chemother 64(11) (2020).

269 [5] M.Y. Jung, J.H. Kang, D.M. Hernandez, X. Yin, M. Andrianifahanana, Y. Wang, A.

270 Gonzalez-Guerrico, A.H. Limper, R. Lupu, E.B. Leof, Fatty acid synthase is required for

271 profibrotic TGF-beta signaling, FASEB J 32(7) (2018) 3803-3815.

272 [6] X. Yin, M. Choudhury, J.H. Kang, K.J. Schaefbauer, M.Y. Jung, M. Andrianifahanana,

273 D.M. Hernandez, E.B. Leof, Hexokinase 2 couples glycolysis with the profibrotic actions of

274 TGF-beta, Sci Signal 12(612) (2019).

275 [7] J.H. Kang, M.Y. Jung, M. Choudhury, E.B. Leof, Transforming growth factor beta induces

276 fibroblasts to express and release the immunomodulatory protein PD-L1 into extracellular

277 vesicles, FASEB J 34(2) (2020) 2213-2226.

278 [8] C.F. Thomas, Jr., A.H. Limper, Current insights into the biology and pathogenesis of

279 Pneumocystis pneumonia, Nat Rev Microbiol 5(4) (2007) 298-308. 
[9] H.M. Evans, A. Simpson, S. Shen, A.J. Stromberg, C.L. Pickett, B.A. Garvy, The Trophic

281 Life Cycle Stage of the Opportunistic Fungal Pathogen Pneumocystis murina Hinders the 282 Ability of Dendritic Cells To Stimulate CD4(+) T Cell Responses, Infect Immun 85(10) (2017).

283 [10] G. Kutty, A.S. Davis, G.A. Ferreyra, J. Qiu, W. Huang da, M. Sassi, L. Bishop, G.

284 Handley, B. Sherman, R. Lempicki, J.A. Kovacs, beta-Glucans Are Masked but Contribute

285 to Pulmonary Inflammation During Pneumocystis Pneumonia, J Infect Dis 214(5) (2016) 782-

28691.

287 [11] M.J. Linke, A. Ashbaugh, M.S. Collins, K. Lynch, M.T. Cushion, Characterization of a 288 distinct host response profile to Pneumocystis murina asci during clearance of pneumocystis 289 pneumonia, Infect Immun 81(3) (2013) 984-95.

290 [12] S.A. Bozzette, F.R. Sattler, J. Chiu, A.W. Wu, D. Gluckstein, C. Kemper, A. Bartok, J.

291 Niosi, I. Abramson, J. Coffman, et al., A controlled trial of early adjunctive treatment with

292 corticosteroids for Pneumocystis carinii pneumonia in the acquired immunodeficiency

293 syndrome. California Collaborative Treatment Group, N Engl J Med 323(21) (1990) 1451-7.

294 [13] R.B. Weyant, D. Kabbani, K. Doucette, C. Lau, C. Cervera, Pneumocystis jirovecii: a 295 review with a focus on prevention and treatment, Expert Opin Pharmacother (2021) 1-14.

297 Supplementary Materials:

298 "Preclinical and Toxicology Studies of BRD5529, a Selective Inhibitor of CARD9"

299 TJ Kottom, AH Limper, et al.

300 Supplementary Table 1

\begin{tabular}{|c|c|c|}
\hline Gene Name & Forward primer & Reverse primer \\
\hline Col1a1 & ATGGATTCCCGTTCGAGTACG & TCAGCTGGATAGCGACATCG \\
\hline$F n$ & GGGTCAGTCCTACAAGATTG & TACAGTCCACCATGAYCCAGCC \\
\hline$B 2 M$ & CTCGGTGACCCTGGTCTTTC & GGATTTCAATGTGAGGCGGG \\
\hline
\end{tabular}

